\title{
Confidence Based Gating of Multiple Face Authentication Experts
}

\author{
Mohammad T. Sadeghi ${ }^{1,2}$ and Josef Kittler ${ }^{2}$ \\ ${ }^{1}$ Signal Processing Research Lab., Department of Electronics \\ University of Yazd, Yazd, Iran \\ ${ }^{2}$ Centre for Vision, Speech and Signal Processing \\ School of Electronics and Physical Sciences \\ University of Surrey, Guildford GU2 7XH, UK \\ \{M.Sadeghi, J.Kittler\}@surrey.ac.uk
}

\begin{abstract}
We address the problem of fusing experts employing diverse similarity measures in LDA face space. The gradient direction measure is reviewed and experimentally compared with the normalised correlation in two different conditions, when the face images are well registered and when the registration process is performed automatically. We show that by combining the gradient direction measure and normalised correlation using a confidence based gating, the resulting decision making scheme consistently outperforms the best method. The gating is based on a novel decision confidence measure proposed in the paper.
\end{abstract}

\section{Introduction}

In certain pattern recognition applications the training sets are notoriously small. A typical example is biometric person recognition where only a few training data points are available for each individual. An extreme case of the small sample set situation arises in image and video database retrieval, where only a single exemplar is available to define the class of objects of interest.

The usual approach to such problems is to base the decision making on some form of similarity measure, or scoring function, which relates unknown patterns to the query object template. If the degree of similarity exceeds a prespecified threshold, the unknown pattern is accepted to be the same as the query object. Otherwise it is rejected. The similarity concept can also be used in recognition scenarios where the unknown pattern would be associated with that class, the template of which is the most similar to the observed data.

The similarity score is computed in a suitable feature space. Commonly, similarity would be quantised in terms of a distance function, on the grounds that similar patterns will lie physically close to each other. Thus smaller the distance, the greater the similarity of two entities. The role of the feature space in similarity measurement is multifold. First of all the feature space is selected so as to maximise the discriminatory information content of the data projected into the feature space and to remove any redundancy. However, additional benefits sought after from mapping the original pattern data into a feature space is to 
simplify the similarity measure deployed for decision making. A classical example of this is the use of the Euclidean distance metric in Linear Discriminant Analysis (LDA) feature spaces as the within class covariance matrix in the LDA space becomes an identity matrix and such metric becomes theoretically optimal. LDA was introduced to the face verification area by Belhumeur in 1996 [1]. Despite the theoretical optimality of Euclidean metric in the LDA space, in [3] , it has been demonstrated that it is outperformed by the Normalised Correlation (NC).

However, in 3 it has been further demonstrated that the Gradient Direction (GD) scoring function is even more effective. In this method the distance between a probe image and a model is measured in the gradient direction of the aposteriori probability of the hypothesised client identity. A mixture of Gaussian distributions with Identity covariance matrix has been assumed as the density function of the possible impostors. In [5] the GD metric was further generalised by considering a general covariance matrix for the components of the Gaussian mixture model(GGD metric). The main problem with the Gradient Direction metric is its computational complexity. In [4, an approximation to the Gradient Direction metric (AGD) was developed. The AGD metric is defined as the difference between the mean (template) of the claimed identity and the local mean of other identities representing the anti-class (impostors). Although not as powerful as the Gradient Direction method, we showed that this approximate Gradient Direction metric gives good performance, in comparison with normalised correlation and is significantly simpler to implement than the Gradient Direction metric method.

The previous studies were performed on the BANCA database 11 using an internationally agreed experimental protocols by applying a geometric face registration method based on manually annotated eyes positions. One of the main issues for assessing the performance of a similarity measure in an automatic face authentication system is how robust the approach is to miss-registration errors. In this paper, the performance of the NC scoring function is compared with the GD metric and its extensions in experiments involving automatically registered faces. Our experimental studies show that overall the $\mathrm{NC}$ function is less sensitive to miss-registration error but in certain conditions GD metric performs better. In order to gain maximum benefit from the complementary merits of these scoring functions, we propose a combined strategy which fuses the scores using a confidence function based weighting. In the proposed method, in the training stage the statistical distribution of the miss-classified scores is estimated for both NC and GD metrics. Then in the test stage, the confidence of the resulting scores are measured. The score value with the higher confidence level is finally adopted for decision making.

The paper is organised as follows. In the next section the Normalised Correlation and Gradient Direction metrics are reviewed. The proposed method of score fusion is then introduced in Section 3. A description of the experimental design including the face database used in the study, the experimental protocols

$\overline{{ }^{1} \text { http://www }} \cdot$ ee.surrey.ac.uk/banca/ 
and the experimental setup is given in Section 4. The experimental results using different scoring functions and the fusion results are presented and discussed in Section 5. Finally a summary of the main findings and conclusions can be found in Section 6.

\section{Similarity Score Functions}

In a face verification system, a matching scheme measures the similarity or distance of the test sample, $\mathbf{x}$ to the template of the claimed identity, $\boldsymbol{\mu}_{i}$. Note that $\mathbf{x}$ and $\boldsymbol{\mu}_{i}$ are the projections of the test sample and class mean into the feature space respectively. The simplest similarity measure, $s$, for matching the probe and the $i$ th client mean is the Euclidean Distance between the vectors $\mathbf{x}$ and $\boldsymbol{\mu}_{i}$, i.e.

$$
s_{E}=\sqrt{\left(\mathbf{x}-\boldsymbol{\mu}_{i}\right)^{T}\left(\mathbf{x}-\boldsymbol{\mu}_{i}\right)}
$$

In [3], it has been demonstrated that a matching score based on Normalised Correlation (NC) scoring function, defined by Equation 2, is more efficient.

$$
s_{N}=\frac{\left\|\mathbf{x}^{T} \boldsymbol{\mu}_{i}\right\|}{\sqrt{\mathbf{x}^{T} \mathbf{x} \boldsymbol{\mu}_{i}^{T} \boldsymbol{\mu}_{i}}}
$$

In [3] an innovate metric called the Gradient Direction (GD) metric has been proposed. In this method the distance between a probe image and a model is measured in the gradient direction of the aposteriori probability of the hypothesised client identity. A mixture of Gaussian distributions with Identity covariance matrix has been assumed as the density function of the possible classes of identity. In [5], we revisited the theory of the Gradient Direction metric and extended it to a Generalised Gradient Direction metric. We demonstrated that applying GD metric using either a general covariance matrix derived from the training data or an isotropic covariance matrix with a variance of the order of the variation of the image data in the feature space is even more efficient than the NC function. The proposed optimal matching score is defined as

$$
s_{O}=\frac{\left\|\left(\mathbf{x}-\boldsymbol{\mu}_{i}\right)^{T} \nabla_{O} P(i \mid \mathbf{x})\right\|}{\left\|\nabla_{O} P(i \mid \mathbf{x})\right\|}
$$

where $\nabla_{O} P(i \mid \mathbf{x})$ refers to the gradient direction. In the generalised form of the GD metric, the optimal direction would be

$$
\nabla_{G} P(i \mid \mathbf{x})=\mathbf{\Sigma}^{-1} \sum_{\substack{j=1 \\ j \neq i}}^{m} p(\mathbf{x} \mid j)\left(\boldsymbol{\mu}_{j}-\boldsymbol{\mu}_{i}\right)
$$


where $p(\mathbf{x} \mid j)$ is the $j$-th client measurement distribution. Considering an isotropic structure for the covariance matrix, i.e. $\boldsymbol{\Sigma}=\sigma \mathbf{I}$, equation 4 could be simplified as:

$$
\nabla_{I} P(i \mid \mathbf{x})=\sum_{\substack{j=1 \\ j \neq i}}^{m} p(\mathbf{x} \mid j)\left(\boldsymbol{\mu}_{j}-\boldsymbol{\mu}_{i}\right)
$$

Note that the magnitude of the $\sigma$ will affect the direction through the values of $p(\mathbf{x} \mid j)$.

\section{Selecting Similarity Functions}

One of the most exciting research directions in the field of pattern recognition and computer vision is classifier fusion. It has been recognised that the classical approach to designing a pattern recognition system which focuses on finding the best classifier has a serious drawback. Any complementary discriminatory information that other classifiers may capture is not tapped. Multiple expert fusion aims to make use of many different designs to improve the classification performance. In the case considered here, as different metrics span the feature space in different ways, it seems reasonable to expect that a better performance could be obtained by combining the resulting classifiers. Our experimental study (reported in section 5) demonstrates that in most of the cases just one of the NC and GD metrics fails to make the correct decision. Therefore, we expect that by dynamically selecting the experts using the respective metrics the performance of the verification system can be improved. In this study, a simple method for combining the NC based classifier and the GD metric one is proposed.

Suppose that $s_{N}$ and $s_{O}$ refer to the NC and GD scores for a test sample, $x$. Let $p_{e}(s)$ denote probability of error. Then, if $p_{e}\left(s_{N}\right)<p_{e}\left(s_{O}\right)$, the NC metric should be used, otherwise the GD metric will give a better result. Now,

$$
p_{e}(s)=p_{e}(s \mid C) p_{e}(C)+p_{e}(s \mid I) p_{e}(I)
$$

where $p_{e}(s \mid C) / p_{e}(s \mid I)$ refers to the probability of error if $x$ is classified as client/impostor and $p_{e}(C)$ and $p_{e}(I)$ refer to the probability of client and impostor errors respectively.

In the evaluation step, in addition to the threshold(s), the probability density functions of the distances corresponding to the miss-classified samples,$P_{e}(s \mid C)$ and $P_{e}(s \mid I)$, can be estimated. These functions are determined for both $\mathrm{NC}$ and GD metrics. In this study a simple unimodal Gaussian function was used for modelling the density functions. $p_{e}(C)$ and $p_{e}(I)$ are in fact the False Rejection and False Acceptance error in the evaluation step. Then, in the test step, the error probabilities of the measured distances are calculated using Equation 6 for both metrics. These values are considered as the confidence levels of the measurements made. The value with the higher confidence level (lower error probability) is used finally to make the decision. 


\section{Experimental Design}

In this section the face verification experiments carried out on images of the BANCA database are described. The BANCA database is briefly introduced first. The main specifications of the experimental setup are then presented.

\subsection{BANCA Database}

The BANCA database has been designed in order to test multi-modal identity verification systems deploying different cameras in different scenarios (Controlled, Degraded and Adverse). The database has been recorded in several languages in different countries. Our experiments were performed on the English section of the database. Each section contains 52 subjects (26 males and 26 females). Experiments can be performed on each group separately.

Each subject participated to 12 recording sessions in different conditions and with different cameras. Sessions 1-4 contain data under Controlled conditions whereas sessions 5-8 and 9-12 contain Degraded and Adverse scenarios respectively. Each session contains two recordings per subject, a true client access and an informed impostor attack. For the face image database, 5 frontal face images have been extracted from each video recording, which are supposed to be used as client images and 5 impostor ones. In order to create more independent experiments, images in each session have been divided into two groups of 26 subjects (13 males and 13 females). Thus, considering the subjects' gender, each session can be divided into 4 groups. The decision function can be trained using only 5 client images per person from the same group and all client images from the other groups.

In the BANCA protocol, 7 different distinct experimental configurations have been specified, namely, Matched Controlled (MC), Matched Degraded (MD), Matched Adverse (MA), Unmatched Degraded (UD), Unmatched Adverse (UA), Pooled test $(\mathrm{P})$ and Grand test $(\mathrm{G})$.

\subsection{Experimental Setup}

The performance of different decision making methods based on the Normalised Correlation $\left(s_{N}\right)$ and the Gradient Direction $\left(s_{I}\right)$ metrics are experimentally evaluated on the BANCA database using the configurations discussed in the previous section. The evaluation is performed in the LDA space. The original resolution of the image data is $720 \times 576$. The experiments were performed with a relatively low resolution face images, namely $64 \times 49$. The results reported in this article have been obtained by applying a geometric face normalisation based on the eyes positions. The eyes positions were localised either manually or automatically. A fast method of face detection and eyes localisation was used for the automatic localisation of eyes centre 22. The XM2VTS database 2 was used for calculating the LDA projection matrix.

$\overline{2}$ http://www.ee.surrey.ac.uk/Research/VSSP/xm2vtsdb/ 
The thresholds in the decision making system have been determined based on the Equal Error Rate criterion, i.e. by the operating point where the false rejection rate (FRR) is equal to the false acceptance rate (FAR). The thresholds are set either globally $(G T)$ or using the client specific thresholding $(C S T)$ technique [5]. As we mentioned earlier, in the training sessions of the BANCA database 5 client images per person are available. In the case of global thresholding method, all these images are used for training the clients template. The other group data is then used to set the threshold. In the case of the client specific thresholding strategy, only two images are used for the template training and the other three along with the other group data are used to determine the thresholds. Moreover, in order to increase the number of data used for training and to take the errors of the geometric normalisation into account, 24 additional face images per each image were generated by perturbing the location of the eyes position around the annotated positions.

In the previous studies [5] 4], it was demonstrated that the Client Specific Thresholding (CST) technique was superior in the matched scenario (Mc, Md, $\mathrm{Ma}$ and G) whereas the Global Thresholding (GT) method gives a better performance on the unmatched protocols. The results reported in the next section were acquired using this criterion.

\section{Experimental Results and Discussion}

Tables 1 contains a summary of the results obtained on the test set when manually annotated eyes position were used for the face geometric normalisation. The values in the table indicate the FAR, FRR and Total Error Rates (TER), i.e. the sum of false rejection and false acceptance rates. In the GD metric the impostor distributions have been approximated by isotropic Gaussian functions with a standard deviation, $\sigma$, of the order of $10^{4}$. The order of $\sigma$ is related to the order of the standard deviation of the input data (gray level values). This order is the consequence of normalising the length of the LDA axes to unity.

Table 1. ID verification results using the Normalised Correlation and Gradient Direction methods with Global and Client Specific Thresholding techniques for unmatched and matched protocols respectively. FAR: False Acceptance Rate, FRR: False Rejection Rate and TER: Total Error Rate.

\begin{tabular}{|c||c|c|c||c|c|c|}
\hline \multicolumn{1}{|c||}{} & \multicolumn{3}{c||}{ NC } & \multicolumn{3}{c|}{ GD } \\
\cline { 2 - 7 } & FAR & FRR & TER & FAR & FRR & TER \\
\hline MC & 2.98 & 5.77 & 8.75 & 1.25 & 3.97 & $\mathbf{5 . 2 2}$ \\
\hline MD & 4.14 & 8.20 & 12.34 & 1.25 & 7.05 & $\mathbf{8 . 3 0}$ \\
\hline MA & 5.96 & 10.00 & 15.96 & 1.35 & 6.53 & $\mathbf{7 . 8 8}$ \\
\hline UD & 13.65 & 13.72 & $\mathbf{2 7 . 3 7}$ & 13.94 & 15.26 & 29.2 \\
\hline UA & 20.19 & 21.92 & 42.12 & 16.06 & 16.15 & $\mathbf{3 2 . 2 1}$ \\
\hline P & 14.01 & 14.23 & 28.24 & 11.57 & 10.64 & $\mathbf{2 2 . 2 1}$ \\
\hline G & 8.20 & 3.33 & 11.54 & 2.02 & 1.58 & $\mathbf{3 . 6 0}$ \\
\hline
\end{tabular}


Table 2. ID verification results using the Normalised Correlation and Gradient Direction methods with Global and Client Specific Thresholding techniques for unmatched and matched protocols respectively. Eyes were localised automatically for the face registration purpose.

\begin{tabular}{|c||c|c|c||c|c|c|}
\hline \multicolumn{1}{|c||}{} & \multicolumn{3}{c||}{ NC } & \multicolumn{3}{c|}{ GD } \\
\cline { 2 - 7 } & FAR & FRR & TER & FAR & FRR & TER \\
\hline MC & 5.096 & 10.9 & $\mathbf{1 6 . 0 0}$ & 8.558 & 8.333 & 16.89 \\
\hline MD & 10.38 & 14.1 & $\mathbf{2 4 . 4 9}$ & 12.98 & 14.1 & 27.08 \\
\hline MA & 10.67 & 10.9 & 21.57 & 11.92 & 9.103 & $\mathbf{2 1 . 0 3}$ \\
\hline UD & 18.85 & 24.62 & $\mathbf{4 3 . 4 6}$ & 23.65 & 23.59 & 47.24 \\
\hline UA & 24.13 & 24.49 & 48.62 & 23.08 & 21.41 & $\mathbf{4 4 . 4 9}$ \\
\hline P & 19.36 & 20.09 & 39.44 & 19.33 & 19.1 & $\mathbf{3 8 . 4 3}$ \\
\hline G & 16.47 & 10.3 & 26.77 & 14.87 & 9.316 & $\mathbf{2 4 . 1 9}$ \\
\hline
\end{tabular}

A comparison of the $\mathrm{NC}$ results against the results using the GD metric in Table 1 clearly demonstrates that the GD metric outperforms the NC metric.

As we mentioned earlier one of the most important criteria for adopting a similarity measure is the robustness of the method against miss-registration errors. In spite of the significant advances in face detection and localisation algorithms, the success of the methods in systems operating in realistic, dynamic scenarios is still very limited. The face pose variation, illumination changes and the size of the face images can degrade the performance of the face localisation algorithms. Table 2 contains the results of similar experiments when the face registration step was performed based on automatically localised eyes position 2].

These results demonstrate that unlike the results using manually localised eyes position, the GD method is not the outright winner in the automatic face verification system. In most of the cases, the NC metric gives a better or comparable verification rate i.e. overall the NC metric seems slightly less sensitive to errors in face registration. In the next section, it is demonstrated that by combining the NC and GD based classifiers, the performance of the face verification system can be improved.

Figure 1 shows a summary of a statistical study of the False Acceptance and False Rejection errors using the NC and GD metrics. In these plots $B F$ and $O F$, respectively, stand for Both metrics Fail and One (and only one) metric Fail. These results demonstrate that, in most of the cases just one of the metrics fails to make the correct decision. Therefore, we expect that by combining the NCbased and GD-based classifiers the performance of the verification system can be improved.

We adopted the decision level fusion strategy proposed in section 3 in order to combine the NC and GD metrics. Tables 3 and 4 contain the combined verification results using manually and automatically registered data respectively. These results demonstrate that, overall, a better performance is achieved using the combined method especially on the unmatched scenarios. The main reason that not much better results are obtained for the matched protocols is that as 


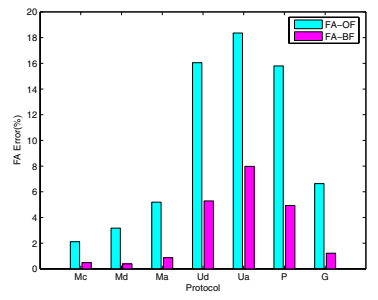

(a) FA error (Manual registration)

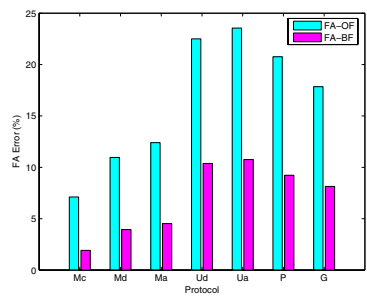

(c) FA error (Automatic registration)

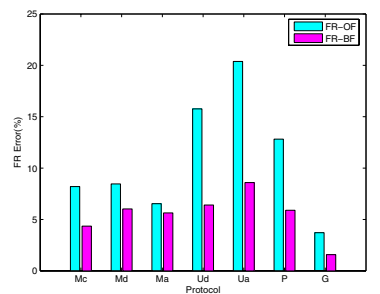

(b) FR error (Manual registration)

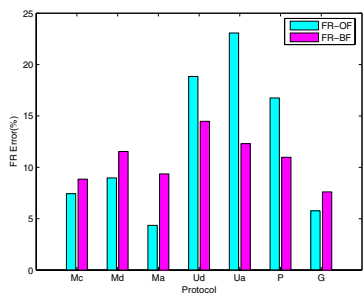

(d) FA error (Automatic registration)

Fig. 1. Percentage of the False Acceptance and False Rejection errors when only One (OF) or Both $(\mathrm{BF})$ metrics fail to make the correct decision

Table 3. ID verification results on BANCA protocols using CST method for matched and GT method for unmatched protocols, manual registration, combining NC and GD scores

\begin{tabular}{||c||c|c|c||c|c|c||}
\hline \multicolumn{1}{||c||}{} & \multicolumn{3}{c||}{ Evaluation } & \multicolumn{3}{c||}{ Test } \\
\cline { 2 - 7 } & FAR & FRR & TER & FAR & FRR & TER \\
\hline Mc & 0.2811 & 0.2821 & 0.5631 & 1.538 & 4.231 & 5.769 \\
\hline $\mathrm{Md}$ & 1.739 & 1.128 & 2.867 & 1.827 & 7.436 & 9.263 \\
\hline $\mathrm{Ma}$ & 1.317 & 0.5641 & 1.881 & 2.5 & 6.667 & 9.167 \\
\hline $\mathrm{Ud}$ & 12.69 & 12.56 & 25.26 & 9.423 & 14.1 & 23.53 \\
\hline $\mathrm{Ua}$ & 19.23 & 19.74 & 38.97 & 14.81 & 17.44 & 32.24 \\
\hline $\mathrm{P}$ & 14.01 & 13.89 & 27.9 & 8.365 & 12.22 & 20.59 \\
\hline $\mathrm{G}$ & 2.387 & 1.111 & 3.498 & 3.59 & 1.41 & 5 \\
\hline
\end{tabular}

we mentioned earlier we adopted the CST technique for these protocols. We only have a few clients per subject and in some cases in the evaluation stage all the clients for each subject are successfully classified. So, there is not enough data available for estimating the distribution of the miss-classified client distance values, $P_{e}(s \mid C)$. Therefore, in the CST method, although the thresholds are client specific, only two global models were estimated as $P_{e}(s \mid C)$ and $P_{e}(s \mid I)$ using the miss-classified samples in the evaluation stage. These models are not as representative as required. 
Table 4. ID verification results on BANCA protocols using CST method for matched and GT method for unmatched protocols, automatic registration, combining $\mathrm{NC}$ and GD metrics

\begin{tabular}{||c||c|c|c||c|c|c||}
\hline \multicolumn{1}{||c||}{} & \multicolumn{3}{c||}{ Evaluation } & \multicolumn{3}{c||}{ Test } \\
\cline { 2 - 7 } & FAR & FRR & TER & FAR & FRR & TER \\
\hline Mc & 0.391 & 0.7436 & 1.135 & 4.808 & 10.38 & 15.19 \\
\hline $\mathrm{Md}$ & 1.154 & 2.077 & 3.231 & 7.885 & 14.62 & 22.5 \\
\hline $\mathrm{Ma}$ & 1.513 & 1.846 & 3.359 & 10.87 & 10.64 & 21.51 \\
\hline Ud & 19.04 & 19.62 & 38.65 & 18.37 & 23.97 & 42.34 \\
\hline Ua & 25 & 25 & 50 & 20.87 & 23.21 & 44.07 \\
\hline $\mathrm{P}$ & 20.13 & 19.74 & 39.87 & 17.44 & 19.4 & 36.84 \\
\hline $\mathrm{G}$ & 2.302 & 3.077 & 5.379 & 14.23 & 11.03 & 25.26 \\
\hline
\end{tabular}

\section{Conclusions}

The problem of measuring similarity in LDA face space has been considered. First, recently proposed gradient direction measures were reviewed and experimentally compared with normalised correlation. The experiments were conducted on the Banca database, using the standard Banca face verification protocols. Although the gradient direction measures were shown to be significantly more discriminative than normalised correlation when probe face images were well registered, in poor registration conditions normalised correlation performed better. As the extent of misregistration was largely a function of the imaging conditions in which the probe data was acquired, the best performing method was effectively scenario dependent.

We showed that by combining the gradient direction measure and normalised correlation using a confidence based gating, the resulting decision making scheme consistently outperformed the best method. The gating is based on a novel decision confidence measure proposed in the paper. The measure, developed in the Bayesian framework, involves estimating the probability distribution of errors for each similarity measure on the evaluation set. The proposed scheme has the advantage that it renders the verification process fully scenario independent.

\section{References}

1. P.N. Belhumeur, J.P. Hespanha, and D.J. Kriegman. Eigenfaces vs. Fisherfaces: Recognition using class specific linear projection. IEEE Trans. on Pattern Recognition and Machine Intelligence, 19(7):711-720, 1997.

2. M. Hamouz, J. Kittler, J.-K Kamarainen, P. Paalanen, H. Kalviainen, and J. Matas. Feature-based affine-invariant localization of faces. IEEE Transactions on Pattern Analysis and Machine Intelligence, 27(9):1490-1495, September 2005.

3. J. Kittler, Y. P. Li, and J. Matas. On matching scores for LDA-based face verification. In M Mirmehdi and B Thomas, editors, Proceedings of British Machine Vision Conference 2000, pages 42-51, 2000. 
4. J. Kittler and M. Sadeghi. Approximate gradient direction metric for face authentication. In Joint IAPR International Workshops on Syntactical and Structural Pattern Recognition and Statistical Pattern Recognition, S+SSPR 2004, pages 797-805, Lisbon, Portugal, 18-20 August 2004.

5. M. Sadeghi and J. Kittler. Decision making in the LDA space: Generalised gradient direction metric. In the 6th International Conference on Automatic Face and Gesture Recognition, pages 248-253, Seoul, Korea, May 2004. 